\title{
LINEAR GROUPS ANALOGOUS TO PERMUTATION GROUPS
}

\author{
W. J. WONG \\ (received 25 October 1962)
}

If $G$ is a finite linear group of degree $n$, that is, a finite group of automorphisms of an $n$-dimensional complex vector space (or, equivalently, a finite group of non-singular matrices of order $n$ with complex coefficients), I shall say that $G$ is a quasi-permutation group if the trace of every element of $G$ is a non-negative rational integer. The reason for this terminology is that, if $G$ is a permutation group of degree $n$, its elements, considered as acting on the elements of a basis of an $n$-dimensional complex vector space $V$, induce automorphisms of $V$ forming a group isomorphic to $G$. The trace of the automorphism corresponding to an element $x$ of $G$ is equal to the number of letters left fixed by $x$, and so is a non-negative integer. Thus, a permutation group of degree $n$ has a representation as a quasi-permutation group of degree $n$.

It seems reasonable to ask whether properties of permutation groups carry over to quasi-permutation groups. In this note I show the validity for quasi-permutation groups of the following simple properties of permutation groups $G$ of degree $n$ :

(i) The order $|G|$ is a divisor of $n$ !

(ii) If $p$ is a prime number exceeding $\sqrt{ } n$, then the Sylow $p$-group of $G$ is of elementary Abelian type.

First, a result on roots of unity.

LEMMA 1. Suppose that the rational integer $s$ is a sum of $n p^{a}$-th roots of 1 , where $p$ is a prime number. Then,

(i) each primitive p-th root of 1 occurs the same number of times;

(ii) if $s \geqq 0$, then the root 1 occurs at least as many times as any primitive $p$-th root of 1 ;

(iii) the number of roots occurring which are not p-th roots of 1 is a multiple of $p$; and

(iv) $s \equiv n(\bmod p)$.

Proor. Let the $p^{a}$-th roots of 1 be

$$
1, \omega, \omega^{2}, \cdots, \omega^{p^{a}-1} \text {. }
$$

Then, the primitive $p$-th roots of 1 are $\omega^{t}, \omega^{2 t}, \cdots, \omega^{(p-1) t}$, where $t=p^{a-1}$. 
Therefore, the following $t$ linear dependence relations amongst the roots hold:

$$
\omega^{k}+\omega^{t+k}+\omega^{2 t+k}+\cdots+\omega^{(p-1) t+k}=0 \quad(k=0,1, \cdots, t-1) .
$$

Now, if $Q$ denotes the rational field, the roots $1, \omega, \omega^{2}, \cdots \operatorname{span} Q(\omega)$ as a vector space over $Q$. Since this vector space has dimension $p^{\alpha-1}(p-1)=$ $p^{a}-t[2$, pp. 112, 162], there can be just $t$ linearly independent linear dependence relations amongst the roots with coefficients in $Q$. Since the relations (1) are clearly linearly independent, every linear dependence relation amongst the roots, with coefficients in $Q$, is a linear combination of the relations (1).

Now suppose that

$$
s=a_{0}+a_{1} \omega+a_{2} \omega^{2}+\cdots, \Sigma a_{i}=n .
$$

The dependence relation

$$
a_{0}-s+a_{1} \omega+a_{2} \omega^{2}+\cdots=0
$$

must be a linear combination of the relations (1). Hence,

$$
\begin{aligned}
a_{0}-s & =a_{t}=a_{2 t}=\cdots=a_{(p-1) t}, \\
a_{k}=a_{t+k}=a_{2 t+k} & =\cdots=a_{(p-1) t+k} \quad(k=1, \cdots, t-1) .
\end{aligned}
$$

The first string of equations proves (i) and (ii), and the other shows that $p$ divides the sum of all the $a_{i}$ for which $i$ is not divisible by $t$, that is, that (iii) holds. For (iv),

$$
\begin{aligned}
n=\sum a_{i} & =\left(a_{t}+s\right)+(p-1) a_{t}+p a_{1}+p a_{2}+\cdots+p a_{t-1} \\
& =s(\bmod p) .
\end{aligned}
$$

THEOREM 1. The order of a quasi-permutation group $G$ of degree $n$ is a divisor of $n$ !

Proof. Let $P$ be a Sylow $p$-group of $G$, of order $p^{a}$. If $x \in P$, the trace $\chi(x)$ of $x$ is the sum of $n p^{a}$-th roots of 1 , and so, by Lemma 1 (iv), is of the form $n-r p$, where $r$ is an integer. Since the only $p^{a}$-th root of 1 which has real part as large as 1 is 1 itself, $r \geqq 0$, and $r=0$ only if $x$ is the identity. Since $\chi(x) \geqq 0, r \leqq N$, where $N=[n / p]$, the integer part of $n / p$. For $r=0,1, \cdots, N$, let $h_{r}$ be the number of elements of $P$ with trace $n-r p$. For any non-negative integer $q, \chi^{q}$ is a (possibly reducible) character of $P$, and so $[1$, p. 263]

$$
\sum_{x \in G} \chi(x)^{a} \equiv 0\left(\bmod p^{a}\right)
$$

i.e.,

$$
\sum_{r=0}^{N} h_{r}(n-r p)^{a} \equiv 0\left(\bmod p^{a}\right) \quad(q=0,1,2, \cdots) .
$$


This is the case $k=0$ of the congruences

$$
k ! p^{k} \sum_{r=0}^{N-k}\left(\begin{array}{c}
N-r \\
k
\end{array}\right) h_{r}(n-r p)^{q} \equiv 0\left(\bmod p^{a}\right) \quad(q=0,1,2, \cdots) .
$$

If we suppose that these congruences hold for a particular value of $k$, then they can be proved for $k+1$ by multiplying (2) by $n-(N-k) p$ and subtracting from the congruence with $q+1$ in place of $q$. Hence, by induction on $k,(2)$ is valid. The case $k=N, q=0$ gives

$$
N ! p^{N} \equiv 0\left(\bmod p^{a}\right) \text {, }
$$

since $h_{0}=1$. But, $N ! p^{N}=p \cdot 2 p \cdot 3 p \cdots N p$ is a divisor of $n !$, since $N p \leqq n$. Hence $p^{a}$ divides $n$ ! Since this holds for all prime divisors $p$ of $|G|$, we have the result.

LEMMA 2. If $P$ is a Sylow p-group of a quasi-permutation group $G$ of degree $n$ smaller than $p^{2}$, then no element of $P$ has order exceeding $p$.

Proof. Suppose if possible that $P$ has an element $x$ of order $p^{2}$. The trace $\chi(x)$ is a sum of $p^{2}$-th roots of 1 . By Lemma 1 (iii), the number of primitive $p^{2}$-th roots of 1 occurring is a multiple of $p$. Hence, the number of primitive $p$-th roots of 1 occurring in $\chi\left(x^{p}\right)$ is a multiple of $p$. But, by Lemma 1 (i), the $p-1$ primitive $p$-th roots of 1 all occur in $\chi\left(x^{p}\right)$ the same number of times. Hence each occurs at least $p$ times. But, by Lemma 1 (ii), this implies that the root 1 also occurs at least $p$ times in $\chi\left(x^{p}\right)$, so that the total number of roots occurring is at least $p^{2}$, contradicting the assumption that $n<p^{2}$.

LeMma 3. If $T$ is a permutation group of degree $n$, whose order is a power of a prime number $p$ greater than $\sqrt{ } n$, then $T$ contains a permutation

$$
\left(a_{11} \cdots a_{1 p}\right)\left(a_{21} \cdots a_{2 p}\right) \cdots\left(a_{m 1} \cdots a_{m p}\right)
$$

such that every element of $T$ is of the form

$$
\left(a_{11} \cdots a_{1 p}\right)^{r_{1}}\left(a_{21} \cdots a_{2 p}\right)^{r_{2}} \cdots\left(a_{m 1} \cdots a_{m p}\right)^{r_{m}} .
$$

In particular, $T$ is of elementary Abelian type.

PROOF. If $N=[n / p]$, then, since $p^{2}>n$, the Sylow $p$-group $S_{p}$ of the symmetric group of degree $n$ has order $p^{N}$. The cycles

$$
(k p+1, k p+2, \cdots,(k+1) p) \quad(k=0,1, \cdots, N-1)
$$

clearly generate an elementary Abelian group of order $p^{N}$, which may therefore be taken as $S_{p}$. Since $T$ must be similar to a subgroup of $S_{p}$, it follows that $T$ is elementary Abelian and that the letters moved by $T$ can be arranged in an order $a_{11}, \cdots, a_{1 p}, a_{21}, \cdots, a_{m p}$, such that every element of $T$ is of the form (3). 
Suppose that $x$ is an element of $T$ moving as many letters as possible:

$$
x=\left(a_{11} \cdots a_{1 p}\right)^{s_{1}}\left(a_{21} \cdots a_{2 p}\right)^{s_{2}} \cdots\left(a_{m 1} \cdots a_{m p}\right)^{s_{m}} \text {. }
$$

Suppose if possible that $s_{1} \equiv 0(\bmod p)$. There exists an element $y$ of $T$ moving $a_{11}$ :

$$
y=\left(a_{11} \cdots a_{1 p}\right)^{t_{1}}\left(a_{21} \cdots a_{2 p}\right)^{t_{2}} \cdots\left(a_{m 1} \cdots a_{m p}\right)^{t_{m}}, t_{1} \not \equiv 0(\bmod p) .
$$

Now, it is possible to choose an integer $k$ such that

$$
k \neq 0(\bmod p) \text {, and }
$$

$$
s_{i}+k t_{i} \neq 0(\bmod p) \text { for all } i \text { such that } s_{i} \neq 0(\bmod p) \text {. }
$$

For, each incongruence is violated by at most one value of $k(\bmod p)$, and there are at most $m$ incongruences. Since $m \leqq n / p<p$, there is a common solution $k$ to all the incongruences. But then $x y^{k}$ moves more letters than $x$, a contradiction. Hence $s_{1} \neq 0(\bmod p)$, and similarly $s_{i} \neq 0(\bmod p)$, all $i$. Replacement of $\left(a_{i 1} \cdots a_{i p}\right)$ by $\left(a_{i 1} \cdots a_{i p}\right)^{s_{i}}$ gives the result.

THEOREM 2. The Sylow p-group of a quasi-permutation group of degree $n$ smaller than $p^{2}$ is of elementary Abelian type.

Proof. Let $P$ be the Sylow $p$-group in question. $P$ may be written as a group of monomial transformations [1, p. 231], that is, there is a basis $e_{1}, \cdots, e_{n}$ of the vector space $V$ on which $P$ acts, such that, for $x$ in $P$, $1 \leqq i \leqq n$,

$$
e_{i}^{x}=a(i, x) e_{j},
$$

where $a(i, x)$ is a scalar, and $j=j(i, x)$ is one of the integers $1, \cdots, n$. For a given $x$, the correspondence $e_{i} \rightarrow e_{j}$ is a permutation $\bar{x}$ of the basis elements, and the set of all $\bar{x}$ forms a permutation group $\bar{P}$ homomorphic to $P$. By Lemma 3, the basis elements can be written as

$$
e_{11}, \cdots, e_{1 p}, e_{21}, \cdots, e_{2 p}, \cdots, e_{m p}, e_{1}, e_{2}, \cdots, e_{r},
$$

in such a way that $\bar{P}$ contains the permutation

$$
\left(e_{11} \cdots e_{1 p}\right)\left(e_{21} \cdots e_{2 p}\right) \cdots\left(e_{m 1} \cdots e_{m p}\right)
$$

and every permutation of $\bar{P}$ is of the form

$$
\left(e_{11} \cdots e_{1 v}\right)^{r_{1}}\left(e_{21} \cdots e_{2 p}\right)^{r_{1}} \cdots\left(e_{m 1} \cdots e_{m p}\right)^{r_{m}} \text {. }
$$

This implies that $V$ is the direct sum of subspaces

$$
V_{1}=\left\{e_{11}, \cdots, e_{m p}\right\}, \quad V_{2}=\left\{e_{1}, \cdots, e_{r}\right\},
$$

each invariant under $P$. For $i=1,2$, let $P_{i}$ be the group of transformations of $V_{i}$ obtained by restricting the transformations in $P$. Since $\bar{P}$ leaves $e_{1}, \cdots, e_{r}$ fixed, $P_{2}$ is Abelian. 
The restrictions to the basis elements of $V_{1}$ of the permutations in $\bar{P}$ form a permutation group $\bar{P}_{1}$ isomorphic with $P_{1} / N$, where $N$ consists of those transformations in $P_{1}$ having $e_{11}, \cdots, e_{m p}$ as eigenvectors. Suppose if possible that $N$ is non-trivial. $P_{1}$ contains an element $x$ such that

$$
e_{i j}^{x}=a_{i j} e_{i, j+1} \quad(i=1, \cdots, m ; j=1, \cdots, p),
$$

where $a_{i j}$ is a scalar, and $e_{i, p+1}=e_{i \mathbf{1}} . N$ is obviously Abelian. By Lemma 2, $N$ is elementary Abelian, and thus may be regarded as a vector space over $G F(p), x$ acts as a linear transformation on $N$, whose minimal polynomial, by Lemma 2 , divides $X^{p}-1=(X-1)^{p}$, and so splits in $G F(p)$. Thus $x$ has an eigenvector in $N$, that is, $x$ commutes with some non-trivial element $y$ of $N$. We have

$$
e_{i j}^{\nu}=b_{i j} e_{i j} \quad(i=1, \cdots, m ; j=1, \cdots, p) .
$$

The fact that $x$ commutes with $y$ implies that

$$
b_{i 1}=b_{i 2}=\cdots=b_{i p} \quad(i=1, \cdots, m) .
$$

Thus each eigenvalue of $y$ occurs with multiplicity at least $p$. But, as in the proof of Lemma 2, no primitive $p$-th root of 1 can occur more than $p-1$ times. Since the eigenvalues of $y$ must be $p$-th roots of 1 (by Lemma 2), they must all be 1 , and this contradicts the fact that $y$ is non-trivial. Hence $N$ must be trivial, and $P_{1}$ is isomorphic with $P_{1}$, which is Abelian, by Lemma 3 .

Since $P_{1}$ and $P_{2}$ are both Abelian, $P$ is itself Abelian, and so elementary Abelian, by Lemma 2 .

Theorems 1 and 2 show that any $p$-group which can be represented as a quasi-permutation group of degree $n$ can also be represented as a permutation group of degree $n$, provided that $p^{2}>n$. The condition is necessary, since, for example, the quaternion group of order 8 has a representation as a quasi-permutation group of degree 4 , but none as a permutation group of degree 4. The investigation of Sylow $p$-groups of quasi-permutation groups, for arbitrary $p$, seems to be difficult.

\section{References}

[1] G. A. Miller, H. F. Blichfeldt and L. E. Dickson, Theory and Applications of Finite Groups (New York, 1938).

[2] B. L. van der Waerden, Modern Algebra, Vol. I (New York, 1949).

University of Otago,

New Zealand. 